The Astrophysical Journal, 222: L33-L36, 1978 May 15

(c) 1978. The American Astronomical Society. All rights reserved. Printed in U.S.A.

\title{
THE BOWEN MECHANISM IN HZ HERCULIS
}

\author{
Bruce Margon* \\ Department of Astronomy, University of California, Los Angeles \\ AND \\ Judith G. CohEN \\ Kitt Peak National Observatory \\ Received 1978 January 9; accepted 1978 February 17
}

\begin{abstract}
Most X-ray star companions show $\lambda 4640$ emission due to an uncertain but probably selective emission process. We have obtained a series of high-dispersion $\left(\sim 14 \AA \mathrm{mm}^{-1}\right)$ blue and ultraviolet spectra of HZ Her, the companion of the X-ray pulsar Her X-1, using the Mayall $4 \mathrm{~m}$ telescope. In addition to the $\mathrm{N}$ III $\lambda 4634-4641$ emission reported by previous observers, the spectra reveal for the first time the presence of $\mathrm{O}$ III $\lambda 3444.1$ emission. This provides conclusive evidence that the Bowen fluorescence mechanism is active in $\mathrm{HZ} \mathrm{Her/Her} \mathrm{X-1,} \mathrm{in} \mathrm{agreement} \mathrm{with} \mathrm{theoretical} \mathrm{predictions} \mathrm{by}$ McClintock et al. and Hatchett et al., and suggests that this is also the correct explanation for the $\lambda 4640$ emission in many such X-ray systems. The observed strengths of the emission features are below those reported in previous lower-dispersion investigations, in some cases very substantially so. The velocities provide no clear resolution of the question of which of the two stars, HZ Her or Her X-1, is associated with the emitting region.

Subject headings: line formation - stars: binaries — stars: individual — X-rays: sources
\end{abstract}

\section{INTRODUCTION}

The optical spectra of the visible counterparts in $\mathrm{X}$-ray binary systems contain substantial information in addition to the data they provide on the velocity of the relatively normal star. For example, they convey valuable insights on the unusual physics of a stellar atmosphere irradiated from above by an intense flux of ionizing photons, sometimes of luminosity orders of magnitude greater than the star itself. Observationally it is found that these spectra often have two properties that probably would not have been predicted in advance: the emission lines are generally weak, even in systems with very high ratios of $\mathrm{X}$-ray to optical luminosity; and lines appear which are apparently due to selective emission processes, as they are too strong to be attributed to nonselective processes, assuming normal abundances. This Letter reports new observations relevant to this latter curious point.

It has been stressed by McClintock, Canizares, and Tarter (1975, hereafter MCT) that most optical counterparts of X-ray sources show a series of weak emission lines in the $\lambda \lambda 4640-4650$ region. Understanding of these lines is complicated by the fact that they are generally faint features in an already faint star, and the exact wavelengths of the emission normally are subject to considerable observational uncertainty. Using a code to simulate an X-ray source surrounded by a uniform distribution of matter, MCT also demonstrated that this emission is quite likely due to a selective process; if the lines are due to $\mathrm{C}$ III, $\mathrm{O}$ II, and $\mathrm{N}$ III transitions

* Visiting Astronomer, Kitt Peak National Observatory, which is operated by the Association of Universities for Research in Astronomy, Inc., under contract with the National Science Foundation. which fall in this region, then the observed strengths are far too large, for example relative to $\mathrm{He}$ II $\lambda 4686$ which is also generally present, to be explained with reasonable abundances. At least two selective processes appear potentially reasonable to explain the observations. The first, favored by MCT, is the Bowen fluorescence mechanism (Bowen 1934, 1935), which generates strong $\mathrm{N}$ III $\lambda 4634-4641$ emission in gaseous nebulae. The second mechanism, also discussed by Davidsen, Margon, and Middleditch (1975), is the "Of star mechanism" (Mihalas 1973; Mihalas and Hummer 1973), which overpopulates the relevant upper levels through a dielectronic recombination from low-lying autoionizing states.

In a simple analysis, both models have several evident virtues and disadvantages. The Bowen process requires an abundant supply of $\mathrm{He}$ II $\mathrm{L} \alpha$ photons ( $\lambda 304)$, whose wavelength coincidence with $\mathrm{O}$ III ultraviolet resonance lines is ultimately responsible for the $\lambda 4640$ emission. In many regions of an X-ray irradiated atmosphere, this condition would seem possible to fulfill, assuming that the geometry is such that photoionization of $\mathrm{H} \mathrm{I}$ and $\mathrm{He} \mathrm{I}$ does not sap the photon supply prior to reaching the line emission region. On the other hand, regardless of the controversy over whether the $\lambda 4640$ lines in HZ Her originate from near the X-ray source (Crampton and Hutchings 1974) or the normal star (Koo and Kron 1977), it is clear that the emitting region is many orders of magnitude more dense than in gaseous nebulae. Thus it might be somewhat surprising if the Bowen mechanism, already a rather unlikely coincidence, is efficiently operative over such a wide range of densities.

A simple morphological argument might be stated in 
favor of the dielectronic recombination mechanism: the spectra of Of stars, where this process is the favored one, resemble at least superficially those of many X-ray sources, in that they show weak He II $\lambda 4686$ and C III/ $\mathrm{N}$ III $\lambda \lambda 4640-4650$ emission (e.g., Conti and Alschuler 1971). In these stars the densities of the line forming regions are much closer to those probably relevant for the X-ray systems. It seems likely that dielectronic recombination is responsible for the $\lambda 4640$ emission in those X-ray systems where the primary has Of star characteristics, e.g., HD 153919 (4U 1700-37). Davidsen, Margon, and Middleditch (1975) presented a simple but quantitative calculation which concluded that dielectronic recombination was also sufficient to explain the observed $\lambda 4640$ line strengths in HZ Her; using somewhat different parameters, MCT reached the opposite conclusion.

Finally, Hatchett, Buff, and McCray (1976) have recently presented an analysis of considerable sophistication of the problem of the transfer of X-rays through a spherically symmetric gas. They conclude, in agreement with MCT, that the Bowen mechanism is likely to be the dominant process responsible for the enhancement $\lambda 4640$ emission in X-ray systems. A rather unambiguous observational test of these theoretical conclusions was suggested by MCT: if the Bowen process is operative, a series of $\mathrm{O}$ III emission lines in the region $\lambda \lambda 3100-3800$ should also be present (cf. Table 4.13 in Osterbrock 1974). Unfortunately, as noted by MCT, the systems in which this emission should be strongest, namely those with large X-ray to optical luminosity ratios, are also the most difficult to observe, especially in the near-UV, due to their faintness. We report here a successful search for these ultraviolet $\mathrm{O}$ III Bowen lines in $\mathrm{HZ}$ Her. The measured line velocities are also relevant to the current controversy concerning the location of the line emitting region in $\mathrm{HZ} \mathrm{Her.}$

\section{OBSERVATIONS AND RESULTS}

The data discussed here were obtained with the $4 \mathrm{~m}$ Mayall telescope of the Kitt Peak National Observatory in 1977 May and August. The May observations utilized the Singer camera on the echelle spectrograph, with a cooled two-stage RCA image intensifier. The 58.5 groove $\mathrm{mm}^{-1}$ echelle yields a dispersion of $13 \AA$ $\mathrm{mm}^{-1}$; IIIa-J plates were used with a projected slit width of $50 \mu \mathrm{m}$, and the spectra are essentially untrailed. The details of these spectra, denoted by SI numbers, are given in Table 1. The August observations utilized the Ritchey-Chrétien spectrograph for the first telescope testing of the recently completed ultraviolettransmitting Simmons camera, with an uncooled twostage RCA image intensifier. A 632 line $\mathrm{mm}^{-1}$ grating blazed at $11000 \AA$ was used in the third order, yielding a dispersion of $14.7 \AA \mathrm{mm}^{-1}$. A $50 \mu \mathrm{m}$ projected slit was used, and the spectra were widened to $0.3 \mathrm{~mm}$. These spectra, denoted by $\mathrm{RC}$ numbers, are also detailed in Table 1.

In both runs, data were obtained in the 3350-3800 and 4300-4800 $\AA$ regions. Phase coverage was purposely biased toward $\phi \sim 0.5$ (maximum light) in the 1.7 day binary period of $\mathrm{HZ}$ Her, as the primary objective was to detect weak emission features. Although our coverage is therefore very nonuniform compared to previous spectroscopic studies of this star, our dispersion of $\sim 14 \AA \mathrm{mm}^{-1}$ quite substantially exceeds that used in the previous extensive studies by Crampton and Hutchings (1974) and Koo and Kron (1977); the latter workers used material of $120 \AA \mathrm{mm}^{-1}$ and $43 \AA \mathrm{mm}^{-1}$, respectively.

An example of our most interesting results is given in Figure 1 (Plate L2), where portions of spectra RC 1408 and RC 1409 are reproduced. These spectra, obtained 1 hour apart, span binary phases $0.40-0.44$, near in time to maximum light and inferior conjunction of the X-ray source. The blue spectrum shows the $\mathrm{N}$ III $\lambda \lambda 4634$, 4641 emission that has been reported by previous observers. The ultraviolet spectrum very clearly displays emission due to $\mathrm{O}$ III $\lambda 3444.1$, reported here, we believe, for the first time. This line is also quite clearly detected on spectrum RC 1395 , obtained 2 days earlier at binary phase 0.285 . These two spectra are the best quality of all our UV material; thus the absence of the $O$ III lines on our other spectra in this wavelength range is consistent with the poorer quality of the

TABLE 1

Journal of Observations of HZ Herculis

\begin{tabular}{|c|c|c|c|c|c|c|c|}
\hline \multirow[b]{2}{*}{ Plate } & \multirow{2}{*}{$\begin{array}{c}\text { JD CENTER } \\
(2,443,000+)\end{array}$} & \multirow[b]{2}{*}{$\phi^{*}$} & \multicolumn{5}{|c|}{ Equivalent Width of Emission (mÅ) } \\
\hline & & & O III 3444.1 & O III 3759.9 & $\mathrm{~N}$ III 4634.2 & $\mathrm{~N}$ III 4641.4 & He II 4686 \\
\hline SI $1674 \ldots \ldots \ldots$ & 290.674 & 0.476 & $\dagger$ & $\ddagger$ & .. & 190 & 110 \\
\hline SI $1676 \ldots \ldots$ & 290.858 & 0.584 & $t$ & $\ddagger$ & & & \\
\hline SI $1687 \ldots \ldots$ & 295.752 & 0.462 & $t$ & $\ddagger$ & 290 & 420 & $<100$ \\
\hline SI $1688 \ldots \ldots$ & 295.780 & 0.479 & $t$ & $\ddagger$ & 310 & 530 & 130 \\
\hline SI $1689 \ldots \ldots$ & 295.798 & 0.490 & $\dagger$ & $\stackrel{\ddagger}{\ddagger}$ & 360 & 480 & $<210$ \\
\hline SI $1690 \ldots \ldots$ & 295.878 & 0.537 & $t$ & $\stackrel{+}{+}+$ & $\ldots$ & $\ldots$ & \\
\hline RC $1394 \ldots \ldots$ & 361.724 & 0.266 & & + & $\cdots$ & $\cdots$ & $\ldots$ \\
\hline RC $1395 \ldots \ldots$ & 361.756 & 0.285 & $500:$ & $\ldots$ & $i$ & $t$ & $t$ \\
\hline RC $1408 \ldots \ldots$ & 363.667 & 0.409 & 346 & $<75$ & $t$ & $t$ & $t$ \\
\hline RC $1409 \ldots .$. & 363.703 & 0.430 & $\dagger$ & $t$ & 220 & 180: & 100 \\
\hline
\end{tabular}

* Phase in 1.7 day binary period; $\phi=0.5$ is maximum light; elements from Giacconi 1974.

$\dagger$ Appropriate spectral region not covered on plate.

$\ddagger$ Plate too weakly exposed to measure emission feature. 
PLATE L2

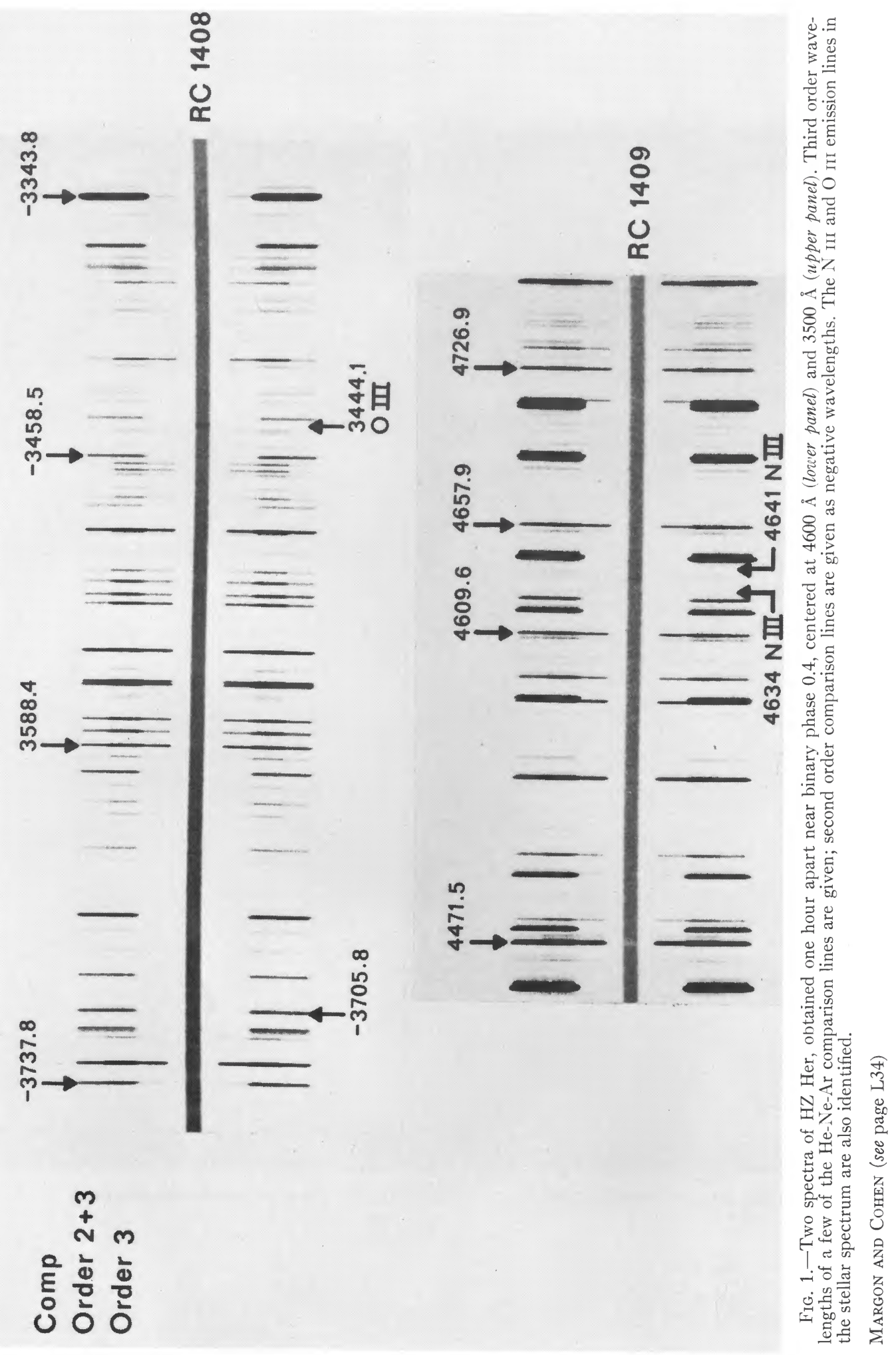


exposures, rather than implying evidence for secular variability.

As predicted by MCT, our detection of the ultraviolet $\mathrm{O}$ III emission provides rather conclusive proof that the Bowen fluorescence mechanism is in fact responsible for the $\lambda 4640$ emission in $\mathrm{HZ} \mathrm{Her,} \mathrm{and} \mathrm{by}$ analogy perhaps also in other $\mathrm{X}$-ray stars. It is of interest to compare the observed ratios of Bowen line strengths in HZ Her to those seen in gaseous nebulae, the only previous astrophysical environment in which this process is confidently observed. To complete this calculation, we must convert our observed equivalent widths of $300-500 \mathrm{~m} \AA$ into absolute fluxes. We have accomplished this conversion through normalization to the continuum multichannel spectrophotometry of Oke (1976) at the appropriate binary phases. As the light curve of HZ Her repeats only to an accuracy of a few tenths of a magnitude on different orbital cycles, we introduce an uncertainty of this order through use of these nonsimultaneous fluxes; however, we are already limited to at least a similar accuracy by our ability to measure the widths of these very weak features, even on the excellent $4 \mathrm{~m}$ plates. The results of this conversion are as follows. We find for the ratio $f(4634) / f(4641)$ in $\mathrm{HZ}$ Her a value of 0.65 . The values observed in the nebulae NGC 7009 and NGC 7662 are summarized by MCT, and imply an analogous ratio of 0.4 . For the value $f(3444) / f(4641)$ we find $\sim 6$ for HZ Her, and $\sim 2$ for the nebulae. The one remaining reasonably strong and accessible Bowen line within our wavelength range is $\mathrm{O}$ III $\lambda 3759.9$. We do not detect this line, to a limit of $75 \mathrm{~m} \AA$ equivalent width, implying $f(3760) /(4641)<1.5$; the value of the ratio for nebulae is 0.5 . Thus the observed Bowen line strengths in HZ Her are in crude agreement with those seen in nebulae. More sophisticated data on this interesting quantity might be obtained through careful narrow-band photoelectric spectrophotometry, which for these weak features in this faint object would be a formidable but feasible task.

The absolute strengths of the Bowen emission features are also of interest because they are a measure of the incident $\lambda 304$ flux, i.e., each $\lambda 304$ photon is ultimately converted to $\lambda 4641$ (MCT; Hatchett, Buff, and McCray 1976). The observed strength of $\lambda 4641$ in our spectra corresponds to $2 \times 10^{-14}$ ergs $\mathrm{cm}^{-2} \mathrm{~s}^{-1}$; the cautions above regarding the systematic calibration uncertainties are also applicable here.

For completeness we have tabulated the observed equivalent widths of the Bowen emission lines, as well as He II $\lambda 4686$ emission, in Table 1 . A few upper limits are also included for the better exposed plates. The strengths we observe for the $\mathrm{N}$ III $\lambda 4640$ lines are somewhat weaker than those reported by $\mathrm{Koo}$ and Kron (1977), and very substantially (up to an order of magnitude) weaker than reported by Crampton and Hutchings (1974) and Hutchings and Crampton (1977). The reason for this discrepancy is not clear, but it is probably not due to a binary phase effect, as both of the previous studies, which have comprehensive phase coverage, agree that there is no systematic variation of $\mathrm{N}$ III strength with phase. The remaining possibilities would seem to be a secular change in the X-ray irradia- tion of the atmosphere, or an instrumental effect due to the very different dispersions used by these three investigations.

The observed velocities of the emission features should of course provide important clues to their point of origin: the disk around the $\mathrm{X}$-ray source, the cool star photosphere, or the stream in between. There is currently no observational consensus on this question, as Crampton and Hutchings (1974) find the emission in approximate antiphase to the HZ Her absorption lines, while Koo and Kron (1977) find rough phase agreement. We had hoped that our substantially better dispersion might resolve this discrepancy, but this is not the case. We adopt for purposes of this analysis the Ca II fitted velocity curve of Koo and Kron (1977) as the elements of HZ Her; as mentioned by these authors, all observers are in reasonably good agreement on these particular data. We have available velocity data only at $\lambda 4634$ and $\lambda 3444$; even at our high dispersion, the blend at $\lambda 4641$ cannot yield reliable information. For these two lines we have data at three binary phases, 0.29 , 0.42 , and 0.48. At these phases the $\mathrm{Ca}$ II elements predict HZ Her velocities of 32,7 , and $-16 \mathrm{~km} \mathrm{~s}^{-1}$, respectively; lines $180^{\circ}$ in antiphase would have velocities $-106,-81$, and $-58 \mathrm{~km} \mathrm{~s}^{-1}$ at these phases. Our observed emission velocities are $-51,-15$, and $4 \mathrm{~km}$ $\mathrm{s}^{-1}$ at the three phases; uncertainties are of order \pm 20 $\mathrm{km} \mathrm{s}^{-1}$. Thus two of our velocities are roughly in phase, while the third is about $90^{\circ}$ out of phase, in agreement with neither of the two previous investigations! We can only conclude that further high-dispersion observations are needed. If the emission remains as weak as we currently measure (cf. Table 1 ), this will be a very challenging problem.

Numerous absorption lines are visible in our spectra, primarily due to the Balmer series, $\mathrm{Ca} \mathrm{I}$ and II, and $\mathrm{Fe} \mathrm{I}$. Their velocities agree with those derived in previous investigations, and so they will not be further considered here. With the exception of the $\mathrm{O}$ III Bowen lines, the ultraviolet spectra are featureless. Many of the blue spectra do show marginal evidence for additional very weak emission features, but we are unable to measure their wavelengths with sufficient accuracy to suggest identifications.

\section{SUMMARY}

Our detection of ultraviolet Bowen emission lines in the spectrum of $\mathrm{HZ}$ Her proves that the Bowen fluorescence mechanism is responsible for the $\lambda 4640$ emission complex, whose origin has until now been vexing. This observation is in agreement with the theoretical predictions of MCT and Hatchett, Buff, and McCray (1976), and should lend considerable confidence that these complex numerical models are in fact correctly describing the X-ray irradiated atmospheres of the primary star in binary X-ray sources. The Bowen fluorescence mechanism is very rarely seen in stellar atmospheres. Haisch et al. (1977) suggest that Bowen $\mathrm{L} \beta$ pumping may explain $\mathrm{O}$ I resonance lines in $\alpha$ Boo, and Oke and Greenstein (1977) propose the presence of O III $\lambda 3444$ in V616 Mon, on the basis of low resolution 
multichannel spectrometer scans. With these possible exceptions, HZ Her is unique in that, whether the emission arises in the cool star photosphere or the disk around the neutron star, the region involved is orders of magnitude more dense than conditions in the gaseous nebulae which Bowen (1934) sought to explain.

One of us (B. M.) acknowledges the financial support of the National Science Foundation.

\section{REFERENCES}

Bowen, I. 1934, Pub. A.S.P., 46, 146. 1935, Ap. J., 81, 1.

Conti, P. S., and Alschuler, W. R. 1971, A p. J., 170, 325.

Crampton, D., and Hutchings, J. B. 1974, A p. J., 191, 483.

Davidsen, A., Margon, B., and Middleditch, J. 1975, A p. J., 198, 653.

Giacconi, R. 1974, in IAU Symposium No. 64, Gravitational Radiation and Gravitational Collapse, ed. C. Dewitt-Morette (Dordrecht: Reidel), p. 147.

Haisch, B. M., Linsky, J. L., Weinstein, A., and Shine, R. A. 1977, Ap. J., 214, 785 .
Hatchett, S., Buff, J., and McCray, R. 1976, Ap. J., 206, 847.

Hutchings, J. B., and Crampton, D. 1977, Astr. Ap., 59, 441

Koo, D. C., and Kron, R. G. 1977, Pub. A.S.P., 89, 285.

McClintock, J. E., Canizares, C. R., and Tarter, C. B. 1975 Ap. J., 198, 641 (MCT).

Mihalas, D. 1973, Pub. A.S.P., 85, 593

Mihalas, D., and Hummer, D. G. 1973, A p. J., 179, 827.

Oke, J. B. 1976, A p. J., 209, 547.

Oke, J. B., and Greenstein, J. L. 1977, A p. J., 211, 872

Osterbrock, D. E. 1974, Astrophysics of Gaseous Nebulae (San Francisco: Freeman).

Judith G. Cohen: Kitt Peak National Observatory, P.O. Box 26732, Tucson, AZ 85726

Bruce MARgon: Department of Astronomy, University of California, Los Angeles, CA 90024 